\title{
Resveratrol Prevented Lipopolysaccharide-Induced Endothelial Dysfunction in Rat Thoracic Aorta Through Increased eNOS Expression
}

\author{
Seda Sultan Uğurel ${ }^{1}$, Nilay Kuşçu ${ }^{2}$, Çiler Çelik Özenci², Selvinaz Dalaklığlu ${ }^{1}$, Arda Taşatargil ${ }^{1}$ \\ ${ }^{1}$ Department of Pharmacology, Akdeniz University School of Medicine, Antalya, Turkey \\ ${ }^{2}$ Department of Histology and Embryology, Akdeniz University School of Medicine, Antalya, Turkey
}

Background: The cardiovascular benefits of Resveratrol (RVT) have been well established by previous experimental and clinical studies.

Aims: The goal of this study was to test the effectiveness of RVT administration on the impaired endothelial function induced by lipopolysaccharide (LPS), and to elucidate the role of endothelial nitric oxide synthase (eNOS)/ Sirtuin 1 (SIRT1) pathway.

Study Design: Animal experiment.

Methods: Endotoxemia was induced by intraperitoneal injection of $10 \mathrm{mg} / \mathrm{kg}$ LPS, and the thoracic aorta was isolated six hours later. RVT was injected intraperitoneally 15 minutes before LPS administration. Six hours after LPS injection, potassium chloride $(\mathrm{KCl})$, phenylephrine (Phe), acetylcholine (ACh), and sodium nitroprusside (SNP) were used to examine to vascular reactivity and endothelial function. eNOS, phospho-eNOS (p-eNOS) (Ser 1177), and SIRT1 expressions in thoracic aorta were evaluated by Western blot.
Results: LPS administration significantly inhibited the relaxation response induced by $\mathrm{ACh}$, while the relaxation to SNP was not significantly altered. Phe- and KClinduced contractile responses in the thoracic aorta significantly decreased in LPS-injected group. eNOS and p-eNOS expression decreased significantly in arteries obtained from LPS group rats. The impaired vasoreactivity as well as decreased expressions of eNOS, p-eNOS, and SIRT1 in vessels from LPS-injected rats were improved by RVT treatment.

Conclusion: The endothelium-dependent vasodilatation of the thoracic aorta was significantly inhibited by LPS administration, and RVT treatment may improve vascular endothelial function. The protective effect of RVT might be associated with increased eNOS expression and activity.

Keywords: Endothelial dysfunction, nitric oxide synthase type III, lipopolysaccharides, resveratrol, Sirtuin 1
Impairment of endothelial function, known as endothelial dysfunction (ED), has been associated with the pathophysiology of sepsis (1), which is one of the most frequent causes of mortality among hospitalized patients. ED is a systemic pathological condition, as defined by a lack of functionally appropriate response of the endothelium in response to a variety of vasodilatory stimuli including shear stress and acetylcholine (2), and has been suggested to be a useful predictor of death from sepsis. Because of the prognostic significance of ED on patient survey, novel therapies are required to prevent the development of ED in sepsis (3).
The promising cardiovascular benefits of the dietary polyphenol Resveratrol (RVT) have been reported by experimental and clinical studies (4). At present, it is known that SIRT1 likely contributes to the regulation of endothelial homeostasis by regulating endothelial nitric oxide synthase (eNOS) (5). An increasing number of studies have reported that RVT, a polyphenolic activator of SIRT1, increases endothelial NO production in endothelial cells by SIRT1-dependent eNOS upregulation. Exposure of cultured endothelial cells for 24-72 $\mathrm{h}$ to RVT increases NO bioavailability via the up-regulation of eNOS mRNA and protein expression (6). Moreover, the treat-

Address for Correspondence: Dr. Arda Taşatargil, Department of Pharmacology, Akdeniz University School of Medicine, Antalya, Turkey. 
ment of human umbilical vein endothelial cells (HUVECs) with RVT for 5 days has been shown to increase the eNOS protein content and NO production (7).

In experimental models, the administration of bacterial lipopolysaccharide (LPS) to animals mimics many of the features of sepsis. RVT may prove to be a therapeutically viable treatment in managing sepsis; however, it is unclear whether acute RVT administration has a potent protective effect on LPS-induced ED.

\section{MATERIALS AND METHODS}

All animal experiments were carried out with the approval of the Animal Experiments Local Ethics Committee. Twelve week-old male Wistar rats (250-300 g) were used in the study. Animals were intraperitoneally injected with either sterile saline of $1 \mathrm{~mL} / \mathrm{kg}$ (Control group) or $1 \mathrm{~mL} / \mathrm{kg} \mathrm{50 \%} \mathrm{ethanol} \mathrm{(Ve-}$ hicle group). The RVT group was intraperitoneally injected with a $20 \mathrm{mg} / \mathrm{kg}$ dose of RVT. The LPS group received a single dose of LPS $(10 \mathrm{mg} / \mathrm{kg})$ intraperitoneally. The LPS+RVT group was pretreated with RVT 15 min before LPS administration. Previous studies have indicated that blood pressure (8), cardiac function $(9,10)$, and vascular reactivity to vasoconstrictors (11) are most significantly changed at this time point.

\section{Isolated organ bath studies}

Six hours after LPS injection, the thoracic aorta from male Wistar rats was rapidly isolated, cut into rings of approximately $3 \mathrm{~mm}$ in length, and mounted in an oxygenated $\left(95 \% \mathrm{O}_{2} / 5 \%\right.$ $\mathrm{CO}_{2}$ ) organ bath with Krebs solution ( $\left.\mathrm{pH} 7.4\right)$ consisting of (millimoles): $\mathrm{NaCl} 118, \mathrm{KCl} 5, \mathrm{NaHCO}_{3} 25, \mathrm{KH}_{2} \mathrm{PO}_{4}$ 1.0, $\mathrm{MgSO}_{4} 1.2, \mathrm{CaCl}_{2} 2.5$, and glucose 11.2. Each ring was connected to a force displacement transducer (FDT10-A, Commat Ltd.; Ankara, Turkey) by using data acquisition unit (MP35, Commat Ltd.; Ankara, Turkey). Resting tension of each vessel ring was set at $2 \mathrm{~g}$ for $60 \mathrm{~min}$. Twelve thoracic aorta rings were used for each group. After the equilibration period, contractile responses were evaluated by potassium chloride $(\mathrm{KCl}$, 20-80 mM) in thoracic aorta rings. Arterial rings were also contracted with phenylephrine (Phe, $10 \mathrm{nmol} / \mathrm{L}-10 \mu \mathrm{mol} / \mathrm{L}$ ). In addition, endothelium-intact tissues were initially contracted with Phe $(1 \mu \mathrm{mol} / \mathrm{L})$ and when the Phe-induced contraction reached a plateau, acetylcholine (ACh, $1 \mathrm{nmol} / \mathrm{L}-100 \mu \mathrm{mol} / \mathrm{L}$ ) and sodium nitroprusside (SNP, $0.1 \mathrm{nmol} / \mathrm{L}-5 \mu \mathrm{mol} / \mathrm{L}$ ) were added cumulatively to the organ bath. This concentration of Phe was chosen to achieve $80 \%$ of the maximum contraction.

\section{SDS polyacrylamide gel electrophoresis and Western blotting}

After the homogenization of thoracic aorta tissues, the supernatants were collected, and the protein concentrations were determined using a Standard BCA assay. Then, $100 \mu \mathrm{g}$ of each protein sample was loaded into each lane. Samples were subjected to SDS polyacrylamide gel electrophoresis under standard conditions and then transferred onto a PVDF membrane overnight. To evaluate the expression of eNOS, p-eNOS, and SIRT1, the membranes were blocked for $1 \mathrm{~h}$ with $5 \%$ non-fat dry milk and were then incubated for $1 \mathrm{~h}$ at room temperature with the anti-rabbit eNOS (sc-654, Santa Cruz Biotechnology; CA, USA), anti-goat p-eNOS (Ser 1177) (sc-12972, Santa Cruz Biotechnology; CA, USA), and anti-rabbit SIRT1 (sc-15404, Santa Cruz Biotechnology; CA, USA). $\beta$-actin was used as an internal standard. The band intensities were evaluated using Image (NIH; Bethsada, MD, USA), which was used to calculate the density of bands.

\section{Chemicals}

All chemicals were purchased from Sigma Chemical (St. Louis; MO, USA), and were dissolved in distilled water, except for RVT (50\% v/v ethanol).

\section{Statistical Analyses}

All data were expressed as the mean \pm SEM. All values were analyzed with GraphPad Prism 4.0 statistical software (GraphPad; CA, USA) using one-way analysis of variance (ANOVA). Post-hoc comparisons were made using Bonferroni post-test. A $p$ value lower than 0.05 was considered significant.

\section{RESULTS}

\section{Effect of acute RVT treatment on vasoreactivity of thoracic artery in LPS-injected rats}

Figure 1 and Figure 2 demonstrate that contractile responses to $\mathrm{KCl}$ and Phe were significantly decreased in arteries that were obtained from LPS-injected animals. In these rats, both contractions were significantly restored by the administration of RVT 15 min before LPS injection. On the other hand, contractile responses to $\mathrm{KCl}$ and Phe were not significantly affected in vessels obtained from the vehicle or RVT group.

Lipopolysaccharide administration caused a significant inhibition on ACh-induced relaxation response (Figure 3, $\mathrm{p}<0.05) . \mathrm{E}_{\max }$ value of ACh-induced relaxation of aortas decreased from $100.0 \pm 0.0 \%$ to $67.20 \pm 3.52 \%$ following LPS administration. Similarly, the $\mathrm{pD}_{2}$ value for ACh-induced re- 


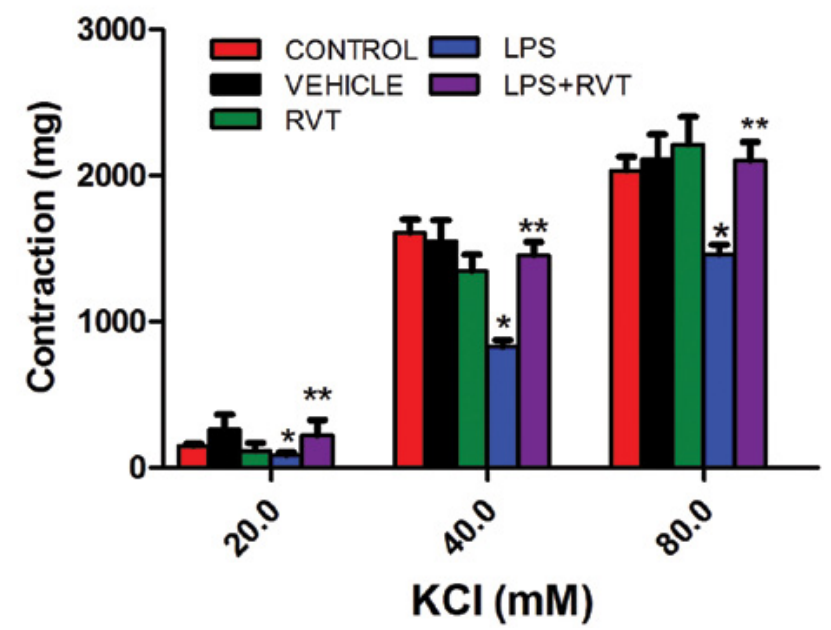

FIG. 1. KCl-induced contraction of rat thoracic aorta in all groups. All values are expressed as mean \pm SEM. $n=12$ for all groups.

(LPS: lipopolysaccharide; RVT: Resveratrol; KCl: potassium chloride)

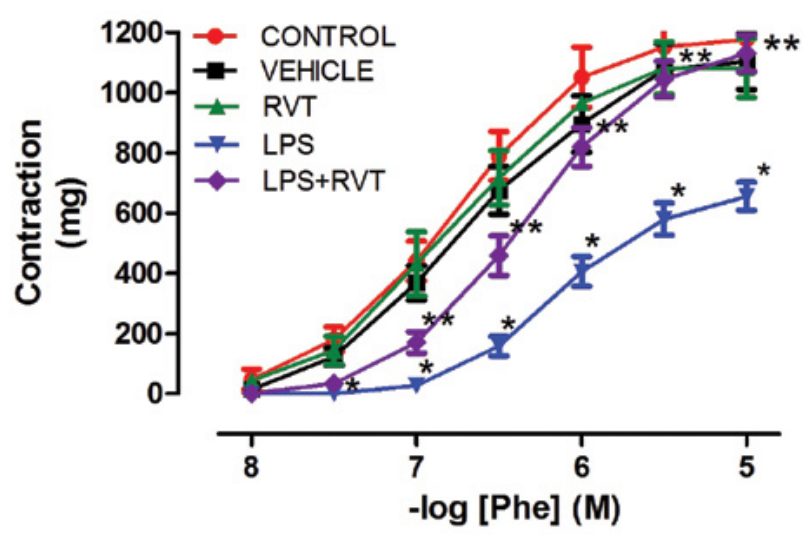

FIG. 2. Phe-induced contraction of rat thoracic aorta in all groups. All values are expressed as mean \pm SEM. $n=12$ for all groups. * $p<0.05$ as compared with controls; ${ }^{* *} p<0.05$ as compared with LPS group.

(LPS: lipopolysaccharide; RVT: Resveratrol; Phe: phenylephrine)

TABLE 1. $\mathrm{pD}_{2}\left(-\log \mathrm{EC}_{50}\right)$ and $\mathrm{E}_{\max }$ values for ACh-induced relaxations of thoracic aorta obtained from control, vehicle, LPS, RVT, and LPS+RVT group rats

\begin{tabular}{lcc}
\hline & $\mathrm{pD}_{2}$ & $\mathrm{E}_{\max }$ \\
\hline Control & $7.91 \pm 0.07$ & $100.00 \pm 0.00$ \\
Vehicle & $7.84 \pm 0.08$ & $100.00 \pm 0.00$ \\
$\mathrm{RVT}$ & $7.90 \pm 0.12$ & $100.00 \pm 0.00$ \\
LPS & $6.70 \pm 0.10^{*}$ & $67.20 \pm 3.52^{*}$ \\
LPS+RVT & $6.98 \pm 0.10^{* *}$ & $96.90 \pm 2.20^{* *}$ \\
\hline
\end{tabular}

All values are expressed as mean \pm SEM. $n=12$ for all groups.

RVT: resveratrol; LPS: lipopolysaccharide; $\mathrm{E}_{\max }$ : maximal response; $\mathrm{ACh}$ acetylcholine chloride

* $\mathrm{p}<0.05$ compared with control, $\mathrm{p}<0.05$ compared with LPS.

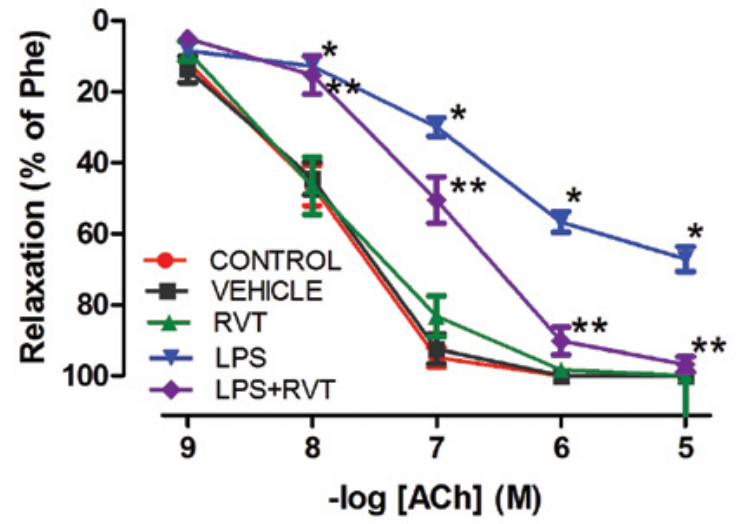

FIG. 3. Endothelium-dependent relaxation responses of thoracic aorta to ACh in all groups. All values are expressed as mean \pm SEM. * $p<0.05$ as compared with controls; ${ }^{* *} p<0.05$ as compared with LPS group. $n=12$ for all groups. (LPS: lipopolysaccharide; RVT: Resveratrol; Phe: phenylephrine; ACh: acetylcholine)

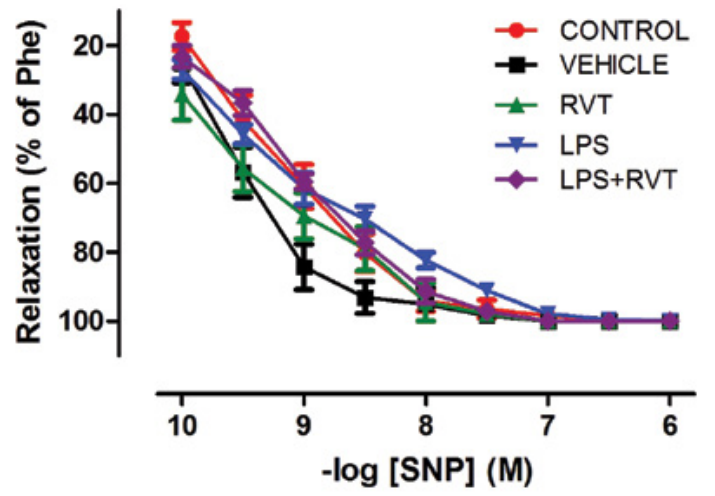

FIG. 4. Endothelium-independent relaxation responses of thoracic aorta to $S N P$ in all groups. All values are expressed as mean $\pm S E M$. $n=12$ for all groups. (LPS: lipopolysaccharide; RVT: Resveratrol; Phe: phenylephrine; SNP: sodium nitroprusside)

laxations was significantly altered after LPS administration (Table 1, $\mathrm{p}<0.05$ ). On the other hand, LPS administration did not cause any significant change in SNP-induced relaxations (Figure 4, $\mathrm{p}<0.05$ ). In LPS-injected rats, the administration of RVT 15 min before LPS injection significantly protected the endothelium-dependent relaxation (Figure 3, Table 1, $\mathrm{p}<0.05$ ). In control rats, however, $\mathrm{ACh}$-induced relaxation response was not significantly changed by acute RVT administration in vivo (Figure 3, Table 1, $\mathrm{p}<0.05$ ).

\section{Effect of acute RVT treatment on eNOS, p-eNOS, and SIRT1 expression in thoracic aorta rings}

In order to evaluate the expression of eNOS, p-eNOS and SIRT1, western blot assays were performed in the aorta; the results are presented in Figure 5. In the control rings, strong eNOS, p-eNOS and SIRT1 expressions were observed. However, a significant reduction in both eNOS and p-eNOS expression was detected in thoracic aorta rings obtained from 
a
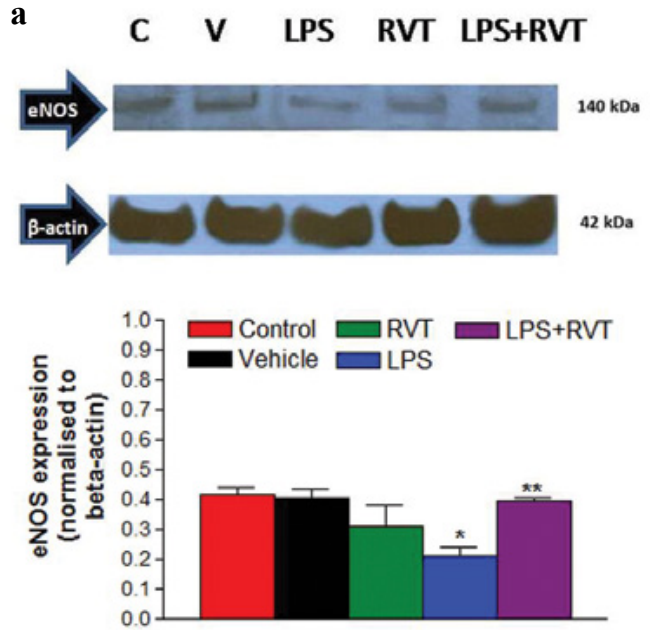

b

\section{V LPS RVT LPS+RVT}
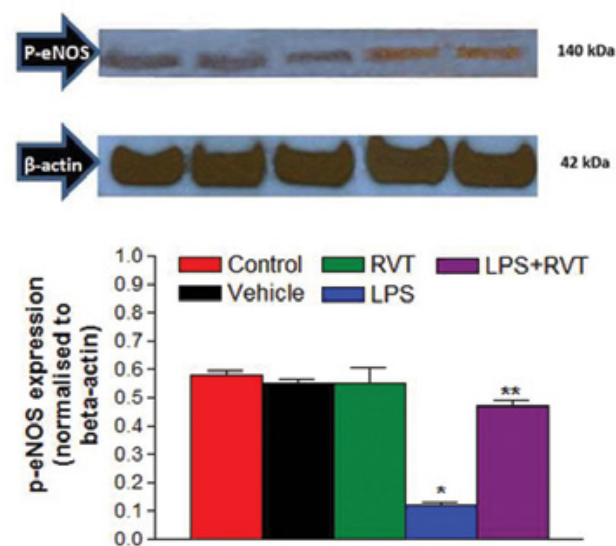

C
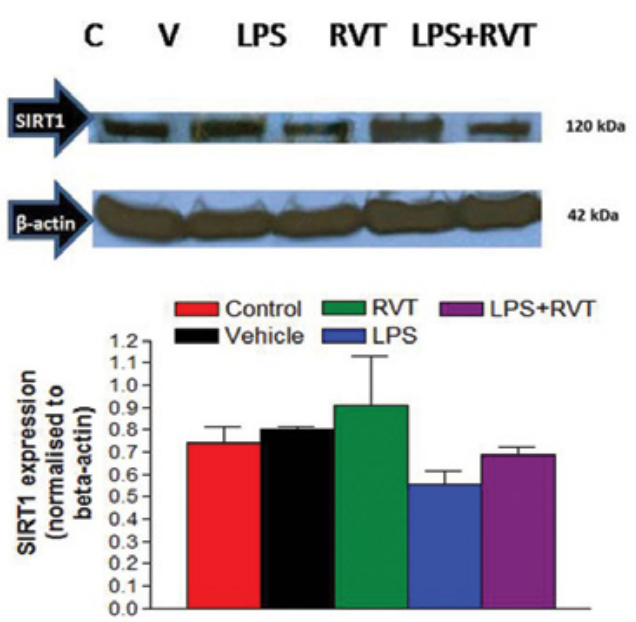

FIG. 5. a-c. Evaluation of eNOS (a), p-eNOS (b) and SIRT1 (c) expressions in thoracic aorta rings by Western blot analysis in all groups. The graphs show image analysis results after Western blot analysis.

${ }^{*} p<0.05$ as compared with controls; ${ }^{* *} p<0.05$ as compared with LPS group. $n=6$ for all groups. (C: control; V: vehicle; LPS: lipopolysaccharide; RVT: Resveratrol; p-ENOS: phosphoendothelial nitric oxide synthase; SIRT1: Sirtuin 1)
LPS-injected rats (Figure 5, $\mathrm{p}<0.05$ ). LPS-related changes in the expression of these proteins were significantly improved by acute RVT treatment. On the other hand, the SIRT1 expression level of aortas obtained from LPS-injected rats was not significantly changed by acute RVT administration.

\section{DISCUSSION}

The goal of this study was to assess the protective effectiveness of RVT administration on endothelial function in LPSinjected rats, and to elucidate eNOS/SIRT1 signaling pathway as a possible mechanism of its protective effect. This study was the first to show that RVT treatment improves endothelium-dependent relaxation of the thoracic aorta in LPS-injected rats primarily via an eNOS-dependent mechanism.

Vascular endothelial cells produce biologically active molecules which regulate blood pressure control. During sepsis, a significant decrease in endothelium-dependent relaxation has been demonstrated in blood vessels from endotoxemic animals $(12,13)$, which is characterized by impaired production of NO by eNOS (14-16). Furthermore, it has been reported that sepsis leads to decreased eNOS phosphorylation in rabbit mesenteric arteries (17). This indicates that the production of NO by eNOS is relatively lower in sepsis. In accordance with these findings, our study clearly demonstrated that LPS administration significantly inhibited endothelial relaxation of the rat thoracic aorta, while the endothelium-independent relaxation response was not affected. Hence, our results indicate that induction of sepsis by LPS causes a detrimental effect, mainly in the endothelium.

The diminished endothelium-dependent relaxation response in LPS-administered rats may be related to the changes in eNOS expression and/or activity. The activity of eNOS is associated with the phosphorylation of serine $1177(18,19)$. The results of the present study indicated that LPS injection caused a significant reduction in eNOS expression and eNOS phosphorylation of the thoracic aorta endothelium. This indicates that a significant reduction in vascular eNOS expression and/ or activity in LPS administered rats may account for the impaired endothelium-dependent relaxation.

Sirtuins may have an important role in the regulation of endothelial homeostasis by regulating eNOS. SIRT1 tends to decline with age, while SIRT1 activators exert significant antiaging impact in the cardiovascular system (20). RVT is the first dietary polyphenol, which has been found to increase SIRT1 activation (21-23). SIRT1 promotes endothelium-dependent relaxation by targeting eNOS deacetylation, thereby enhancing NO production (5). Based on the above explanation, RVT may have a therapeutic potential in managing sepsis, however, there is no study investigating its effect on decreased vaso- 
reactivity in the course of sepsis. Our results indicated that acute administration of RVT to LPS-injected rats efficiently increased endothelium-dependent relaxation induced by ACh. In contrast, RVT did not cause any significant change in the SNP-induced relaxations. In the light of these findings, protective effect of RVT on thoracic aorta relaxation is related with increased NO production of endothelial cells, but not likely due to a RVT-mediated increase in cGMP levels. Also, our results have demonstrated that the administration of RVT improved the eNOS and p-eNOS protein expression levels of the thoracic aorta in LPS-injected rats. The phosphorylation itself was potentiated by RVT, as the ratio of p-eNOS to total eNOS was increased in RVT plus LPS treated rats, when compared with LPS group. Potentiation of eNOS and p-eNOS expression with RVT treatment also paralleled with the improvement of endothelial function. Taken together, this indicates that LPS may cause ED by decreasing the eNOS expression and/or activation, and RVT prevents LPS-induced ED through increasing NO-mediated endothelium-dependent relaxation. The results of Xia et al. have shown that the SIRT1/FOXO axis is an important modulator of enhanced eNOS gene expression and activity induced by RVT (24). Although RVT administration also resulted in an increase in SIRT1 expression, the difference between the LPS and LPS plus RVT groups was not significant.

In conclusion, the present study demonstrates that RVT might prevent the development of ED in the course of sepsis through an eNOS-dependent mechanism. In the future, RVT might be a meaningful treatment for patients with sepsis in whom the NO pathway is impaired.

Ethics Committee Approval: Ethics committee approval was received for this study from the Local Ethics Committee.

\section{Informed Consent: N/A.}

Peer-review: Externally peer-reviewed.

Author contributions: Concept - S.S.U., A.T.; Design - A.T., Ç.Ç.Ö.; Supervision - A.T., S.S.U.; Resource - A.T., Ç.Ç.Ö.; Materials - A.T., S.S.U.; Data Collection and/or Processing - S.S.U., N.K., Ç.Ç.Ö.; Analysis and/or Interpretation - S.S.U., N.K., Ç.Ç.Ö.; Literature Search - S.S.U., A.T.; Writing - S.D., S.S.U., A.T.; Critical Reviews - S.D., A.T.

Conflict of Interest: No conflict of interest was declared by the authors.

Financial Disclosure: This study was supported by Akdeniz University Research Foundation (Grant No. 2011.02.0122.002).

\section{REFERENCES}

1. Peters K, Unger RE, Brunner J, Kirkpatrick CJ. Molecular basis of endothelial dysfunction in sepsis. Cardiovasc Res 2003;60:49-57. [CrossRef]

2. Poredos P. Endothelial dysfunction and cardiovascular disease. Pathophysiol Haemost Thromb 2002;32:274-7. [CrossRef]

3. Aird WC. Endothelium as a therapeutic target in sepsis. Curr Drug Targets 2007;8:501-7. [CrossRef]

4. Wu JM, Wang ZR, Hsieh, TC, Bruder JL, Zou JG, Huang YZ. Mechanism of cardioprotection by resveratrol, a phenolic antioxidantpresent in red wine (Review). Int J Mol Med 2001;8:3-17.

5. Mattagajasingh I, Kim CS, Naqvi A, Yamamori T, Hoffman TA, Jung SB, et al. SIRT1 promotes endothelium-dependent vascular relaxation by activating endothelial nitric oxide synthase. Proc Natl Acad Sci U S A 2007;104:14855-60. [CrossRef]

6. Wallerath T, Deckert G, Ternes T, Anderson H, Li H, Witte K, et al. Resveratrol, a polyphenolic phytoalexin present in red wine, enhances expression and activity of endothelial nitric oxide synthase. Circulation 2002;106:1652-8. [CrossRef]

7. Takahashi S, Nakashima Y. Repeated and long-term treatment with physiological concentrations of resveratrol promotes NO production in vascular endothelial cells. Br J Nutr 2012;107:774-80. [CrossRef]

8. Lalu MM, Csont T, Schulz R. Matrix metalloproteinase activities are altered in the heart and plasma during endotoxemia. Crit Care Med 2004;32:1332-7. [CrossRef]

9. Khadour FH, Panas D, Ferdinandy P, Schulze C, Csont T, Lalu $\mathrm{MM}$, et al. Enhanced NO and superoxide generation in dysfunctional hearts from endotoxemic rats. Am J Physiol Heart Circ Physiol 2002;283:H1108-15. [CrossRef]

10. Lalu MM, Gao CQ, Schulz R. Matrix metalloproteinase inhibitors attenuate endotoxemia induced cardiac dysfunction: a potential role for MMP-9. Mol Cell Biochem 2003;251:61-6. [CrossRef]

11. Lalu MM, Cena J, Chowdhury R, Lam A, Schulz R. Matrix metalloproteinases contribute to endotoxin and interleukin-1beta induced vascular dysfunction. Br J Pharmacol 2006;149:31-42. [CrossRef]

12. Parker JL, Adams HR. Selective inhibition of endothelium-dependent vasodilator capacity by Escherichia coli endotoxemia. Circ Res 1993;72:539-55. [CrossRef]

13. Umans JG, Wylam ME, Samsel RW, Edwards J, Schumacker PT. Effects of endotoxin in vivo on endothelial and smoothmuscle function in rabbit and rat aorta. Am Rev Respir Dis 1993;148:1638-45. [CrossRef]

14. Salzman AL, Wang H, Wollert PS, Vandermeer TJ, Compton CC, Denenberg AG, et al. Endotoxin-induced ileal mucosal hyperpermeability in pigs: role of tissue acidosis. Am J Physiol 1994;266:G633-46.

15. Zhou M, Wang P, Chaudry IH. Endothelial nitric oxide synthase is downregulated during hyperdynamic sepsis. Biochim Biophys Acta 1997;1335:182-90. [CrossRef]

16. Matsuda N, Hattori Y, Zhang XH, Fukui H, Kemmotsu O, Gando S. Contractions to histamine in pulmonary and mesenteric 
arteries from endotoxemic rabbits: Modulation by vascular expressions of inducible nitric-oxide synthase and histamine H1receptors. J Pharmacol Exp Ther 2003;307:175-81. [CrossRef]

17. Matsuda N, Hayashi Y, Takahashi Y, Hattori Y. Phosphorylation of endothelial nitric oxide synthase is diminished in mesenteric arteries from septic rabbits depending on the altered phosphatidylinositol 3-kinase/Akt pathway: Reversal effect of fluvastatin therapy. J Pharmacol Exp Ther 2006;319:1348-54. [CrossRef]

18. Fleming I, Busse R. Molecular mechanisms involved in the regulation of the endothelial nitric oxide synthase. Am J Physiol Regul Integr Comp Physiol 2003;284:R1-12. [CrossRef]

19. Fulton D, Gratton JP, McCabe TJ, Fontana J, Fujio Y, Walsh K, et al. Regulation of endothelium-derived nitric oxide production by the protein kinase Akt. Nature 1999;399:597-601. [CrossRef]

20. Zarzuelo MJ, López-Sepúlveda R, Sánchez M, Romero M, Gómez-Guzmán M, Ungvary Z, et al. SIRT1 inhibits NADPH oxidase activation and protects endothelial function in the rat aorta: implications for vascular aging. Biochem Pharmacol 2013;85:1288-96. [CrossRef]

21. de Boer VC, de Goffau MC, Arts IC, Hollman PC, Keijer J. SIRT1 stimulation by polyphenols is affected by their stability and metabolism. Mech Ageing Dev 2006;127:618-27. [CrossRef]

22. Kaeberlein M, McDonagh T, Heltweg B, Hixon J, Westman EA, Caldwell SD, et al. Substrate-specific activation of sirtuins by resveratrol. J Biol Chem 2005;280:17038-45. [CrossRef]

23. Borra MT, Smith BC, Denu JM. Mechanism of human SIRT1 activation by resveratrol. J Biol Chem 2005;280:17187-95. [CrossRef]

24. Xia N, Strand S, Schlufter F, Siuda D, Reifenberg G, Kleinert H, et al. Role of SIRT1 and FOXO factors in eNOS transcriptional activation by resveratrol. Nitric Oxide 2013;32:29-35. [CrossRef] 